\title{
Automatic Extraction of Symmetry Plane from Falx Cerebri Areas in CT Slices
}

\author{
Darius Grigaitis ${ }^{1}$ and Mecislovas Meilunas ${ }^{2}$ \\ ${ }^{1}$ Department of Electronics Systems \\ ${ }^{2}$ Department of Mathematical Modelling \\ Vilnius Gediminas Technical University, Naugarduko 41, LT-03227, Vilnius, Lithuania \\ Email: darius.grigaitis@el.vtu.lt
}

\begin{abstract}
We present the simple and fast symmetry plain detection algorithm, that recognizes Falx cerebri curve on each human brain computed thomogrpahy slice. Symmetry curves appear approximately on $30 \%$ images and using such images as reference it is possible to determine symmetry plane. We propose an algorithm based on hybrid methods, that allows detect symmetry plane with deviation angle until $25^{\circ}$. The method is based on fuzzy logic that selects region of interest and symmetry curves. Direct pixels selection with evaluation of symmetry curve properties are used to calculate symmetry plane with high speed.
\end{abstract}

\section{Introduction}

Determination of symmetry plane (SP) on human brain images is a crucial task for further automatic analysis. Division of brain region into symmetric areas provides more accuracy in detection of brain structures and recognition of nonhealthy regions of the brain like stroke, aneurism, tumor, etc. There are many methods to compute mid-sagitall symmetry plain of brain. Some of them measure cross correlation $[1,2,3,4]$ or Hough transform [5] or other features [6, 7, 8]. Disadvantage of such algorithms are time-consuming calculations, especially in the case of cross correlation method. Therefore high speed algorithms are required. One of possible ways is to use distributed computing or optimize existing algorithms. In our task the specific fast hybrid principle was used that extracts Falx cerebri feature (Fig. 1). The designed method uses direct point search method in order to increase noise resistance and avoid Hough transform floating point of angle calculations. Because our proposed algorithm works with only integer numbers, the time of calculation is less then 1 second to calculate symmetry plane using 50 images by $512 \times 512$ resolution with Pentium 4, 2.6GHz computer.

\section{Method}

Algorithm consists of four stages. First stage is image preprocessing, definition of the region of interest (ROI). The scope of future analysis is elimination of unnecessary image information for axis determination. The smaller brain view 
Fig. 1. An example of symmetry curve in CT images of this same person: higher slice (left); lower slice (right)
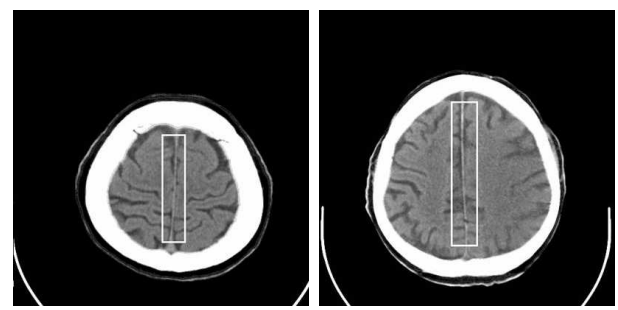

area helps to reach better filter response on symmetry curve (SC). In the second stage filtering is implemented in order to select narrow light curves (Fig. 1) called Falx Cerebri. Almost 30\% CT images contain this information in human vertex area. Third stage is detection of symmetry axis. In this stage SC are converted into the lines in order to calculate SP. Conversion is implemented by simple separation of pixels by lines with some angle [9]. The last stage is related to SP calculation using symmetry lines coordinates detected in previous stage. For correct SP detection minimum two lines must be detected.

\subsection{Definition of the region of interest}

The proposed algorithm requires clean areas to detect SC. However, there are critical areas in CT images that possess properties similar to SC. This is left and right human brain areas, that after processing with SC filter appears as vertical curves. By this reason, left and right areas are removed and speed of SP detection increase, because the area of brain can be reduced more than in $40 \%$. The algorithm executes from the calculation of the center point of brain view and diagonal lines (Fig. 2) that show corresponding areas of brain view in each slice.

The brain region in image is defined as gray valued pixels

$$
S=\{(x, y), g(x, y)>0\}
$$
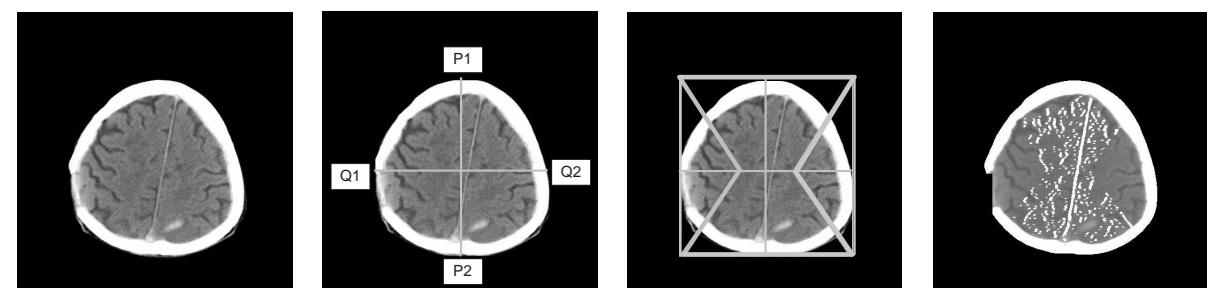

Fig. 2. Region of interest selection: initial image (left); center of object detection (middle left); diagonal lines for area separation (middle right); ROI after filtering (right) 
Here, $x, y$ - coordinates of pixels, $g(x, y)$ - human brain image with bone areas. In the beginning of ROI selection the boundaries of brain view are denoted as follows

$$
\begin{array}{r}
Q_{1}\left(\underline{x}, y_{l}\right), Q_{2}\left(\bar{x}, y_{r}\right) \\
P_{1}\left(x_{b}, \underline{y}\right), P_{2}\left(x_{t}, \bar{y}\right)
\end{array}
$$

Here, $y_{l}, y_{r}$ - left and right pixels of objects in $y$ coordinate, $x_{t}, x_{b}$ - top and bottom pixels of objects in $x$ coordinate $, \bar{x}, \underline{x}, \bar{y}, \underline{y}$ is left, right and top, bottom grey level view pixels positions

$$
\begin{aligned}
& \underline{x}=\min _{i}\left\{x_{i} \mid\left(x_{i}, y_{j}\right)\right\} \in S, \underline{y}=\min _{i}\left\{y_{j} \mid\left(x_{i}, y_{j}\right)\right\} \in S \\
& \bar{x}=\max _{i}\left\{x_{i} \mid\left(x_{i}, y_{j}\right)\right\} \in S, \bar{y}=\max _{i}\left\{y_{j} \mid\left(x_{i}, y_{j}\right)\right\} \in S
\end{aligned}
$$

Using such information any line shown in Fig. 2 can be calculated. The gap between diagonal lines is ROI and SC can easily fit with 25 degrees angle or more. The shape of ROI is not significant, it was only important to remove left and right areas (Fig. 2) that filters recognize as parts of SC.

\subsection{Detection of symmetry curve}

Several types of filters that are related to symmetry curve properties was analyzed. In Fig. 3 is shown filters shapes that are used for analyzing its behavior on symmetry curve (SC). The maximum size of sliding window of filter was $10 \times 10$ pixels because symmetry curve can easily fit in it. The main property of symmetry curve is brighter pixels than other gray matter pixels entire image. Thus, the simple fuzzy filters for brightness comparison were constructed. These filters are suitable to recognize vertical light curves. Construction of tested filters Fig. 3 is shown. The main property of filters is that center pixels (black squares) are compared with surrounding pixels (white squares) by rule

$$
I_{b}(x, y)= \begin{cases}1, & g_{\text {left }}(x, y) \& g_{\text {right }}(x, y)>g_{c}(x, y) \\ 0, & \text { otherwise }\end{cases}
$$

Here, $I_{b}(x, y)$ - center pixels of sliding window of binary images; $g_{c}(x, y)$ - center pixels of sliding window of gray scale images; $g_{\text {left }}(x, y), g_{\text {right }}(x, y)$ - left and right pixels of sliding window of gray scale image.

For testing of filters we used 1462 images of 91 patients. $60 \%$ of slices where eliminated automatically because they have not SC property. As testing parameter the length of SC was used, that was calculated with fourth step of designed algorithm. The experiment showed that histograms of SC length of given filters differ slightly and shape of filter is not essential. Moreover, if the sliding window of filter becomes more simple than Fig. 4, then SC can not be detected, when $\mathrm{SC}$ appears very unclear. There is eventually strong relation between number of comparison in filter, brightness and length of SC pixels (Fig. 2). 
Fig. 3. An example of used filters shapes for SC detection

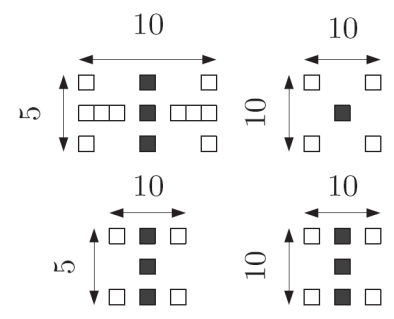

\subsection{The estimation of symmetry axis}

In symmetry curve detection stage, sectors of filtered images are collected into lines $B=\left\{\left(x_{i}, y_{i}\right), g\left(x_{i}, y_{i}\right)>0\right\}$. Here the Hough transform can be used, that can draw the line through the concentrated pixels $[10,5]$. According noisy CT images we used direct point selection by lines with appropriate angle. In Fig. 4 is shown an example of lines positioning. First, two horizontal and parallel lines are used. Second, from the $q_{1}$ the lines are drawn by formula of standard line between two points. All straights positions are stored and used to produce all possible lines between horizontal lines. The number of possible lines is $M=q_{n} \cdot r_{m}$, which has set of pixels $l_{i j}$ where $1 \leq i \leq m, 1 \leq j \leq m$ are set of filtered pixels (Fig. 2). Here $m, n$ - lengths of horizontal lines. In estimation of symmetry line is important intersection of filtered pixels and produced lines $k_{i j}=\sum l_{i j} \cap B$. One of lines can represent symmetry axis in case when meets the requirements of $\left(i_{0}, j_{0}\right)=\arg \max \left(k_{i j}+\delta\right)$. The parameter $\delta=\sum_{s=1}^{k_{i j}} \sum_{p=1}^{P} \sum_{r=1}^{R} \sum_{l=1}^{L}\left(g_{p s}(x, y)-g_{l r}^{p s}(x, y)\right)$ evaluates SC brightness of pixels by their neighbours. This simple addition dramatically increases $k_{i j}$ value and symmetry axis can be detected even SC is represented with low number pixels. In this case the filtered image (Fig. $2 \mathrm{~d}$ ) can contain more noise pixels than SC. Finally symmetry plane equation is derived using $N=2 \mathrm{~K}$ number of points in $30 \%$ slices. Here $K$ is number of CT slices of single patient.

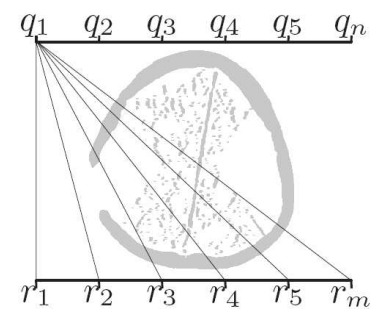

Fig. 4. An example of symmetry curve detection by lines, the bone area was placed to show proper image position 


\section{Results}

Experiments with CT images showed that shape of filter is not essential and simplest filter shape can be used. The critical parameter that affect speed is forming of straights (Fig. 4). If only odd values of $m, n$ are used to draw straights then time of calculation decreases more than 35\%, but precision of SP detection decreases. To speed up an algorithm the straights are calculated once and copied to determine all possible angles between horizontal lines. For experiments we used 1462 CT images of 91 person. The SP were detected successfully for 89 persons. Visually all 89 images collections showed correct position of SC in $-25^{0} . .+25^{0}$ angle interval. Because the symmetry curves appear only in human head vertex area, then symmetry axis are projected in to all slices. This algorithm can be used for magnetic nuclear resonance images also. For precision evaluation of formed SP the future analysis of comparison must be prepared.

\section{References}

1. Prima S, Ourselin S, Ayache N. Computation of the mid-sagittal plane in 3D brain images. IEEE Trans Med Imaging 2002; 122-138.

2. Tuzikov AV, Colliot O, Bloch I. Brain symmetry plane computation in MR images using inertia axes and optimization. Institute of Engineering Cybernetics Academy of Sciences of Republic Belarus: IEEE; 2002. 1051-4651.

3. Ardekani BA, Kershaw J, Braun M, Kanuo I. Automatic detection of the midsagittal plane in 3D brain images. IEEE Trans Med Imaging 1997;16:947-952.

4. Liu Y, Collins RT, Rothfus WE. Robust midsagittal plane extraction from normal and pathological 3D neuroradiology images. IEEE Trans Med Imaging 2001;20:175-192.

5. Brummer ME. Hough transform detection of the longitudinal fissure in tomographic head images. IEEE Trans Med Imaging 1991;10:74 - 81.

6. Anbazhagan P, Carass A, Bazin P, Prince JL. Automatic estimation of midsagittal plane and AC-PC alignment based on nonrigid registration. Procs IEEE Symp Biomed Imaging 2006; 828 - 831.

7. Prima S, Ourselin S, Ayache N. Computation of the mid-sagittal plane in 3D brain images. IEEE Trans Med Imaging 2002;21:122-138.

8. Smith S, Jenkinson M. Accurate robust symmetry estimation. Procs MICCAI 1999; 308-317.

9. Grigaitis D, Zitkevicius E, Usinskas A. Determination of symmetry axis on human brain CT image. Procs Bienlial Baltic Electronics Conf 2004; 165-168.

10. Gonzalez RC, E WoodsR. Digital Image Processing. Prentice-Hall, Inc.- Second Edition; 2002. 